\title{
Lysozyme expression is increased in the sinus mucosa of patients with chronic rhinosinusitis*
}

\author{
Charmaine M. Woods', Vivienne S. Lee', Damian J. Hussey', Shabnam \\ Irandoust ${ }^{2}$, Eng H. Ooi', Lor-Wai Tan ${ }^{3}$ and A. Simon Carney ${ }^{1}$
}

Flinders ENT, Department of Surgery, Flinders University and Flinders Medical Centre, Adelaide, Australia

SA Pathology, Flinders Medical Centre, Adelaide, Australia

Department of ENT Surgery, Queen Elizabeth Hospital, Adelaide University, Adelaide, Australia
Rhinology 50: 147-156, 2012

DOI:10.4193/Rhino11.229

*Received for publication:

October 21, 2011

accepted: January 10, 2012

\section{Summary}

Background: The presence of fungi and bacteria in the paranasal sinuses may contribute to ongoing inflammation. Lysozyme is an innate immune peptide with bactericidal and fungicidal activity. The expression of lysozyme in chronic rhinosinusitis (CRS) is poorly understood and deficiencies in lysozyme expression may contribute to the ongoing inflammation in CRS patients.

Objective: Determine lysozyme expression in sinus mucosa of normal and CRS patients with (CRSwNP) and without (CRSsNP) nasal polyps.

Methodology: Sinus mucosa specimens $(n=82)$ were processed for standard histology, immunohistochemical localisation of lysozyme, immunofluorescent localisation of fungi, and qPCR analysis of lysozyme expression.

Results: CRS specimens displayed high-levels of lysozyme immunoreactivity in many of the abundant serous cells. Moderate levels were detected in some epithelial cells and inflammatory cells. Low levels were detected in some subepithelial glands of control specimens. No difference in immunoreactivity was detected between CRSwNP and CRSsNP specimens. Fungal elements were not visualised in any sinus specimen. QPCR analysis demonstrated variable lysozyme expression between individuals.

Conclusions: Lysozyme protein expression is increased in patients with CRS, suggesting a defect in lysozyme expression is not responsible for the microbial colonisation often associated with CRS. The functional activity of lysozyme in CRS patients needs to be further investigated.

Key words: muramidase, sinusitis, antimicrobial cationic peptides, nasal polyps, antifungal agents

\section{Introduction}

Nasal secretions containing innate immune defence proteins, including lysozyme, lactoferrin, human $\beta$-defensin (HBD) and secretory leukocyte protease inhibitor (SLPI) form an important component of innate immunity against inhaled antigens and micro-organisms ${ }^{(1-3)}$. Lysozyme is the most abundant secreted innate immune defence protein from the paranasal sinuses ${ }^{(4)}$. It is usually regarded as a bactericidal agent, with well-characterised enzymatic activity causing cell lysis by catalysing hydrolysis of a polysaccharide component in the cell wall of gram-positive bacteria ${ }^{(5)}$. However, lysozyme is also a cationic protein and its bactericidal action is not solely dependent on its enzymatic activity ${ }^{(6-8)}$. We recently demonstrated that lysozyme has fungicidal activity towards a number of filamentous fungal species commonly found in patients with chronic rhinosinusitis (CRS) ${ }^{(9)}$. 
Many bacterial species have developed mechanisms to overcome host innate immunity ${ }^{(10,11)}$, and an interesting observation of progressive resistance to the fungicidal activity of lysozyme by yeast in AIDS patients ${ }^{(12)}$ has raised the possibility that fungi may also develop mechanisms capable of evading the host's innate defences. However, we have recently demonstrated in vitro that fungi isolated from the mucin of CRS patients were not resistant to lysozyme ${ }^{(9)}$ and hypothesised that a deficiency in lysozyme mRNA expression and/or protein secretion may be present in CRS patients. Little is known about lysozyme in the paranasal sinuses ${ }^{(13-16)}$ and this study was conceived to determine the expression of lysozyme in the sinus mucosa of normal controls and CRS patients with (CRSwNP) and without (CRSsNP) nasal polyps.

\section{Materials and methods}

\section{Tissue Acquisition}

The protocol for this study was approved by the Flinders Clinical Research Ethics Committee. Patients with CRS as defined by the American Academy of OHNS ${ }^{(17)}$ who failed maximal medical treatment and consented to undergo endoscopic sinus surgery were recruited for the study. Sinus mucosa specimens were collected from CRS patients $(n=76)$ and divided into two groups according to the presence of polyps at surgery ( $n=39$ CRSwNP; $n=37$ CRSsNP). Normal sinus mucosa specimens $(n=6)$ were obtained from patients undergoing endoscopic transphenoidal pituitary surgery without any clinical or radiological evidence of rhinosinusitis.

Two biopsies were collected from each patient. Biopsies destined for gene expression analysis were placed immediately into RNAlater (Ambion, NY, USA) and stored at $-20^{\circ} \mathrm{C}$ until required. The second biopsy was placed in formalin and following standard processes prepared into paraffin blocks for histochemical staining and histopathological evaluation. Blood samples were taken from CRS patients for determination of total IgE and aero-allergen specific lgE (assays performed by commercial pathology laboratories).

\section{Lysozyme mRNA expression experiments}

RNA Extraction and Reverse Transcription

Tissue (20 - $25 \mathrm{mg}$ ) was homogenized in $1000 \mu \mathrm{L}$ of TRIzol ${ }^{\circ}$ (Invitrogen Life Technologies, NY, USA) using a plastic pestle attached to a Dremmel ${ }^{\circ}$ MultiPro $^{\mathrm{TM}}$ drill. Total RNA was extracted according to the manufacturer's protocol, using the recommended modified precipitation step for samples containing proteoglycans and/or polysaccharides. RNA concentration was determined with the NanoDrop 8000 Spectrophotometer (ThermoScientific, Wilmington, DE, USA). RNA quality was determined by electrophoresis through a $1 \%$ agarose gel and confirmed it was not degraded by visualization of distinct
$28 \mathrm{~S}$ and $18 \mathrm{~S}$ rRNA species. Samples containing degraded RNA were excluded from the RT-PCR anaylsis. cDNA was prepared using the Quantitect $\mathrm{RT}^{\mathrm{TM}}$ kit (Qiagen, Germany). DNAse treatment of total RNA (150 ng RNA) was performed prior to reverse transcription according to the manufacturer's protocol. A negative reverse transcriptase control reaction (omission of the reverse transcriptase) was included.

Real-time quantitative Polymerase Chain Reaction The resulting CDNA was diluted 1:2 and PCR amplification was performed in $20 \mu \mathrm{L}$ final volumes containing $3 \mu \mathrm{L}$ cDNA template, $2 \mu \mathrm{L}$ each of forward and reverse primers, $3 \mu \mathrm{L}$ UPW, and $10 \mu \mathrm{L} 2 \times$ Quantitect SYBRGreen Master Mix (Qiagen, Germany). Thermocycling utilized a Rotorgene 6000 cycler (Corbett, Australia) and PCR conditions were the same for the two amplicons. Primer sequences for lysozyme and the housekeeping gene hypoxanthine phosphoribosyl transferase (HPRT $^{(18-20)}$ ) are in Table 1. PCR conditions consisted of an initial denaturation at $95^{\circ} \mathrm{C}$ for $15 \mathrm{~min}$, followed by 45 cycles of denaturation $95^{\circ} \mathrm{C}$ for $20 \mathrm{sec}$, annealing $55^{\circ} \mathrm{C}$ for $30 \mathrm{sec}$ and extension at $72^{\circ} \mathrm{C}$ for $30 \mathrm{sec}$, with a final extension at $72^{\circ} \mathrm{C}$ for $4 \mathrm{~min}$. Triplicate reactions were performed on all samples. A melting curve analysis of the products was performed at the end of each PCR assay and single melting point peaks were identified for the target and reference house-keeping genes. PCR products (lysozyme 196bp, HPRT 93bp) were verified by nucleotide sequencing and BLAST search.

Cycle thresholds were determined using the relative quantification analysis module in the Rotorgene 6000 Series software (Corbett Research, Australia). The amplification efficiency of each primer pair was estimated from a real-time PCR dilution curve generated using serial twofold dilutions of genomic DNA. Quantitative real-time RT-PCR analysis was then performed using Q-Gene software ${ }^{(21)}$. Relative lysozyme expression data was normalized according to the corresponding levels of HPRT for each sample.

\section{Lysozyme protein expression experiments}

Histology

Formalin-fixed, paraffin-embedded tissue sections ( $3 \mu \mathrm{m}$ ) were deparaffinised through three changes of xylene and rehydrated through successive immersion in absolute ethanol and distilled water. Sequential sections were processed and stained with haematoxylin and eosin (H\&E), and periodic acid Schiff with diastase (PASD), then visualised with a brightfield Olympus BX photomicroscope.

\section{Immunohistochemical localisation of lysozyme}

Tissue sections were deparaffinised and rehydrated. Positive lysozyme immunoreactivity in sinus tissues was detected 
following digestion with Proteinase $\mathrm{K}$ (pH 7.5, Dako, CA, USA) for $5 \mathrm{~min}$ at room temperature. Polyclonal rabbit anti-human lysozyme antibody (Dako) was used at 1:900 dilution for 30 min. For a negative control the primary antibody was substituted with rabbit immunoglobulin fraction (dilution 1:900, Dako). Visualisation of lysozyme was achieved with Envision+ system-HRP (AEC) (Dako), to give an insoluble red precipitate. Sections were counterstained with haematoxylin.

The immunostaining of lysozyme was scored by two independent assessors blinded to the category of the patient. Lysozyme immunoreactivity was reported as a visual percentage of the total number of glands or epithelial cells. Staining intensity was scored as 0-3, based on a pre-defined density template.

\section{Immunofluorescent localisation of fungi}

Tissue sections were deparaffinised through three changes of xylene, rehydrated through three changes of absolute ethanol and cleared in successive immersion in acetone through to PBS. Sections were incubated in 5\% periodic acid for $20 \mathrm{~min}$ at room temperature and blocked with $5 \%$ bovine serum albumin prior to application of Fungalse-F (Anomeric, LA, USA), a fluorescein-labelled chitinase stain (1:2 dilution for 30min in the dark). Slides were then washed with two changes of PBS and directly mounted with ProLong Gold containing DAPI as counterstain (Invitrogen). Aspergillus fumigatus grown on agar slopes and double mounted into paraffin blocks were used as positive controls to demonstrate validity of the fungal stain.

Fungalase-F stained slides were examined using the fluorescence Olympus BX50 microscope for specific detection of green (emission 515-555 nm) fluorescing fungi against DAPI stained violet (emission 420-460 nm) fluorescing nuclei.

Particular attention was given to fluorescence occurring in the mucous, along the epithelium and within subepithelial glands. The slides were viewed by two independent assessors blinded to clinical outcome, and reported as presence or absence of fluorescence consistent with the size and shape of fungal hyphae or conidia/spores.

\section{Statistical analysis}

Statistical analyses utilised SPSS 16.0 for Windows software (SPSS, Inc.). Analysis of RT-PCR data involved one-way analysis of variance with Bonferroni correction for multiple comparisons between groups. Analysis of immunohistochemistry utilised the non-parametric Kruskal-Wallis test with adjusted significance for pairwise comparisons. Statistical significance was set at $p<0.05$.

\section{Results}

\section{RT-PCR results}

mRNA expression of lysozyme was conducted in 59 sinus samples (control =6, CRSwNP = 29, and CRSsNP = 24). Lysozyme mRNA was amplified in all sinus specimens. When normalised to HPRT expression, a substantial range in relative lysozyme mRNA expression values was observed (Figure 1A). No statistically significant difference was observed between CRSwNP and CRSsNP ( $p>0.05$, ANOVA). No statistically significant difference was observed between control specimens and specimens from CRS patients with either normal ( $<110 \mathrm{kU} / \mathrm{L})$ or high ( $>110 \mathrm{kU} / \mathrm{L}$ ) serum IgE status (Figure 1B, p > 0.05, ANOVA). Similarly, lysozyme mRNA expression data was analysed according to patients' allergic status and no statistical difference was observed between control specimens and either allergic or non-allergic CRS specimens (Figure 1C, p > 0.05, ANOVA).

\section{Immunohistochemistry results}

H\&E staining of control sinus specimens demonstrated normal histology, whereas CRS specimens displayed signs of acute and/or chronic inflammation often associated with marked tissue eosinophilia. Low-levels of lysozyme-like immunoreactivity were present in some sub-epithelial glands of normal sinus mucosa and epithelial cells (Figure 2A, D, G). In comparison, CRS specimens displayed high-levels of immunoreactivity in many of the abundant seromucous glands, especially the serous cells (Figure 2E, F). Moderate levels were detected in some epithelial cells and in inflammatory cells throughout the tissue of CRS specimens (Figure 2B, C, H, I). Lysozyme-like immunoreactivity was observed in a higher percentage of seromucous glands (Figure 3A, p < 0.05) and epithelial cells (Figure 3B, p < 0.05 ) in CRS specimens compared to normal sinus specimens. In addition the intensity of staining was also greater in the CRS specimens compared to normal samples ( $p<0.05$, Figure $3 C-D)$. There was no statistical difference between the levels of immunoreactivity observed in patients with CRSwNP vs CRSsNP $(p>0.05$, Figure 3$)$.

\section{Fluorescent localisation of fungi}

Fungalase-F clearly identified fungal hyphae and conidia in the positive control specimen containing paraffin embedded colonies of Aspergillus fumigatus (Figure 4A). In comparison the sinus samples displayed autofluorescence of red blood cells and non-specific debris (Figure 4C), making identification of any potential fungal hyphae particularly difficult. Fungal elements were not observed in any CRS or control specimen using the fluorescent Fungalase-F stain, despite increased lysozyme immunoreactivity detected in the sequential section. Similarly, fungal elements were not observed on H\&E or PASD

(Figure 4B) stained sections. 

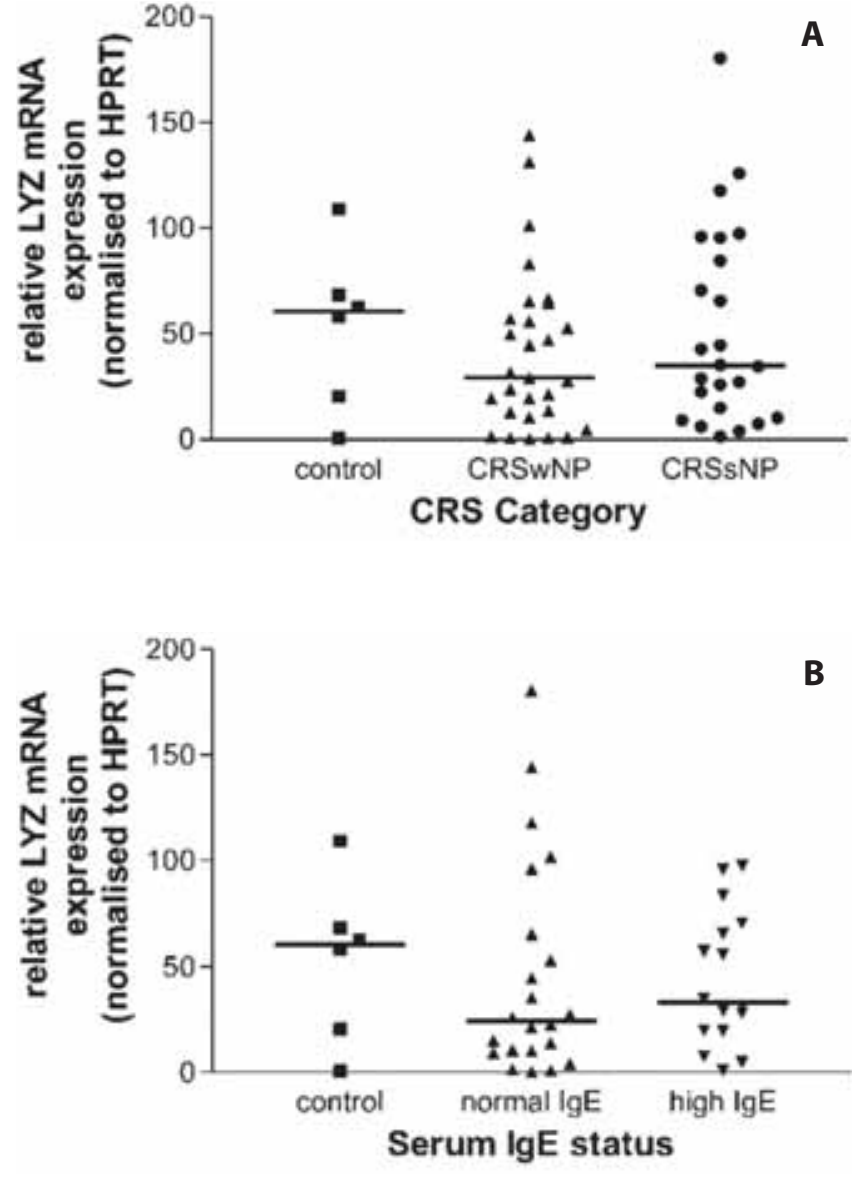

\section{Discussion}

Cationic antimicrobial peptides are important components of innate immunity and increasing evidence indicates that the pathogenesis of CRS may involve a deficiency of the innate immune system thus facilitating bacterial and/or fungal colonisation with chronic inflammation ${ }^{(2)}$. In addition, there is increasing evidence that microbes can develop mechanisms to overcome host innate defences ${ }^{(10,11)}$. The innate peptide lysozyme is abundant in nasal secretions and to date has been characterised as an antibacterial agent with its enzymatic muramidase activity. However, our recent data demonstrated lysozyme has fungicidal activity, supporting a role for lysozyme in paranasal sinus innate immunity against bacterial and fungal colonisation ${ }^{(9)}$. Our recent in vitro investigations suggested that fungi cultured from the mucous of CRS patients were still susceptible to the fungicidal activity of lysozyme. Therefore in this study, we investigated the hypothesis that there is a deficiency in lysozyme production in CRS patients, which could contribute to on-going microbial colonisation.

\section{mRNA expression of lysozyme}

First, we assessed the relative mRNA expression levels of lysozyme in normal, CRSwNP and CRSsNP tissue samples. The relative mRNA expression data failed to demonstrate a

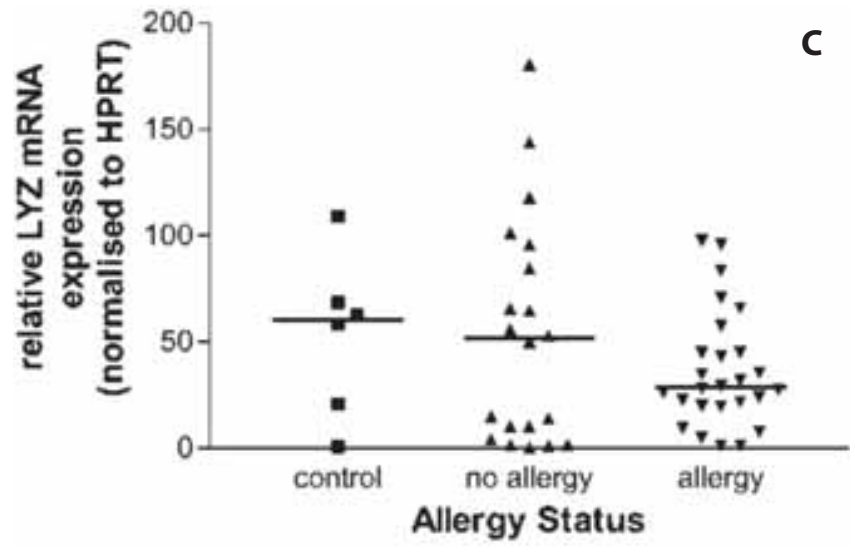

Figure 1. Relative lysozyme mRNA expression in sinus mucosa. Relative lysozyme mRNA expression was normalised to expression of the housekeeping gene HPRT. Data was analysed according CRS status (control, CRSwNP, or CRSsNP; (A), serum IgE status (control, CRS with normal $\lg \mathrm{E}$ or CRS with high $\lg \mathrm{E}$; (B), and allergy status (control, CRS with no allergy, CRS with yes allergy; (C). A substantial range in lysozyme mRNA expression values was observed within each classification group suggesting a high degree of interpersonal variability. No statistical difference was observed between control and CRS classification groups ( $p$ > 0.05 , ANOVA), or IgE status ( $p>0.05$; ANOVA), or allergy status ( $p>0.05$; ANOVA). $\mathrm{N}=6$ control, 29 CRSwNP, 24 CRSsNP, 22 normal lgE, 16 high lgE, 20 no allergy, 26 allergy. Bar indicates group median value.

difference in lysozyme mRNA expression levels between normal and CRS specimens. Due to the considerable variability in lysozyme mRNA values within each group it is not possible to draw any conclusions regarding the regulation of lysozyme in CRS patients, other than to state that variability exists within control, CRSwNP and CRSsNP groups. This may be a result of natural variation, or possibly due to the interaction between the innate and acquired immune systems.

Variability in lysozyme mRNA expression levels has also been detected within epithelial cells from sinus polyps ${ }^{(14)}$ and in healthy small intestinal mucosal samples ${ }^{(22)}$. Lysozyme expression in polyp epithelial cells is similar to the normal inferior turbinate ${ }^{(14)}$. It is possible that some of this variability might be attributed to the effect of transcription factors on lysozyme gene expression. The transcription factor ESE-1b has been found to transactivate the lysozyme promoter, however other ESE isoforms also have differential regulating potentials on the lysozyme promoter ${ }^{(23)}$, which could account for some of the variability observed here. Interestingly, in patients with ulcerative colitis, lysozyme mRNA expression was highly elevated in those with active disease, whereas those with inactive disease had lower levels ${ }^{(22)}$. This suggests that disease severity at the time of tissue collection may be another contributing factor 
to the varying levels observed in the sinus specimens. In this study, although all CRS patients had clinical evidence of sinus disease at surgery there may be other variables (e.g. the presence of an acute allergic phase of seasonal allergic rhinitis) that have influenced the results.

There are few studies investigating lysozyme mRNA expression in sinus tissue. In normal human nasal epithelial cells (NHNE) it was found that the culture process caused significant changes in lysozyme expression compared to cells from ex vivo tissue ${ }^{(24,25)}$. Furthermore, as lysozyme is predominantly found within the serous cells of the submucosal glands and not the epithelial cells (see below), studies utilizing only epithelial cells or NHNE cell culture may not be representative of lysozyme in the paranasal sinuses. To our knowledge, this is the first study to investigate lysozyme mRNA expression in nasal mucosa.

\section{Lysozyme protein distribution}

Protein expression of lysozyme has been determined immunohistochemically in a number of studies. We found that the increase in lysozyme protein distribution was similar between CRSwNP and CRSsNP. Electron microscopy investigations of inferior turbinate tissue identified lysozyme in serous cells of glands of the nasal mucosa ${ }^{(26)}$ and also in the goblet cells of the nasal epithelium (16). An immunohistochemical study using nasal turbinate tissue obtained from patients undergoing turbinectomies for nasal obstruction found lysozyme present in serous cells but not in mucous cells or epithelial goblet cells (15). Our findings in normal sinus specimens are consistent with Fukami et al., (13) where the epithelium was generally lysozyme negative with a weak positive reaction in the goblet cells, and low intensity staining of some serous cells of the subepithelial glands and inflammatory cells throughout the tissue. Likewise, in CRS tissues we also found an increase in the number of positive epithelial cells. However, in contrast to Fukami et al., ${ }^{(13)}$ in tissues obtained from CRS patients there was a considerable increase in the number of lysozyme-positive serous cells within the glands and the intensity of the staining. The increased production of lysoyzme protein suggests that in CRS patients there is an increased and/or constant stimulation of innate immune pathways - perhaps due to increased microbial load (dead or alive, bacteria and/or fungi), or inflammatory signals.

It is important to recognise that the mRNA expression data does not correlate with the protein expression data. There are many theoretical circumstances where mRNA expression does not result in increased protein production and secretion. For example, post-transcriptional regulation by microRNAs can reduce protein production from an mRNA transcript by microRNA sequence binding to complementary mRNA sequence in the 3 prime untranslated region of the mRNA ${ }^{(27)}$.
It is possible that in the absence of sinusitis, microRNAs downregulate lysozyme production and that this barrier is lifted when CRS exists. Changes in microRNA expression patterns are known to be involved in Toll-like receptor signaling ${ }^{(28)}$ and other inflammatory conditions ${ }^{(29)}$ but very little is known about their role in the etiology of CRS. To determine preliminary support for the possibility of regulation of lysozyme production by microRNA we searched for miRNAs predicted to target the lysozyme transcript. We used the miRDB database, which was generated using a training algorithm based on experimental data in order to accurately identify nucleic acid sequence features important for microRNA:mRNA transcript interaction (30). The output revealed a total of 14 microRNAs predicted to target the LYZ transcript, thereby supporting the possibility of lysozyme regulation by microRNAs.

\section{Clinical implications}

Our results would imply that a deficiency in lysozyme production in these CRS patients is not contributing to the on-going microbial (bacterial and/or fungal) colonisation often associated with CRS. We believe that there are two independent factors at play; first increased production of lysozyme protein, and second functional activity of lysozyme protein. The protein is produced in greater amounts due to stimulation of the innate immune pathways. However, one would assume that increased lysozyme protein would result in less microbial infections. Therefore, we now postulate that a deficiency in lysozyme function may be occurring in patients with CRS. An increase in the ionic strength of the surrounding airway surface liquid (ASL) would inhibit the binding of cationic antimicrobial peptides to the anionic microbial cell wall and prevents cell lysis. Chronic microbial colonisation and infection occur in the lungs of cystic fibrosis patients, and the ASL in cystic fibrosis is known to impair the activity of cationic antimicrobial peptides ${ }^{(31,32)}$. A recent study found substantially increased levels of lysozyme and lactoferrin in bacterial infected bronchoalveolar lavage fluid obtained from cystic fibrosis patients suggesting that lysozyme is produced and secreted, but it is inactive. This may also occur in the secretions of CRS patients, especially considering the ASL ionic strength of normal healthy nasal mucosa is believed to be hypotonic ${ }^{(33)}$ - but this is controversial with other studies reporting ASL ion concentrations to be isotonic ${ }^{(34)}$. In comparison, the ASL is reported to be isotonic in patients with sustained airway infection or cystic fibrosis (33). This theory then raises questions regarding the current practice of saline irrigation as a treatment modality for CRS, as the ionic strength may in fact be inhibiting the action of these important innate peptides and could be providing conditions favourable for microbial colonisation. We believe this is a direction requiring further investigation. 
Normal

A
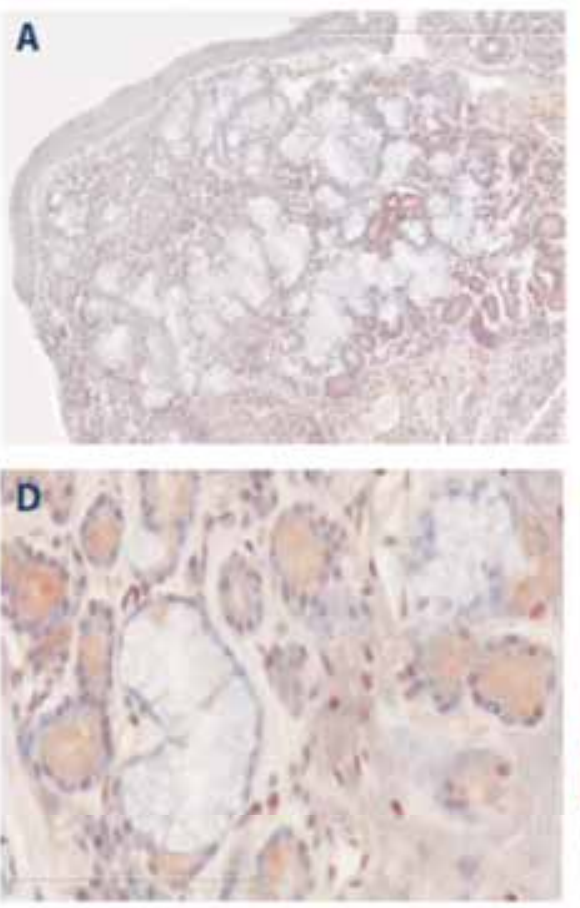

G

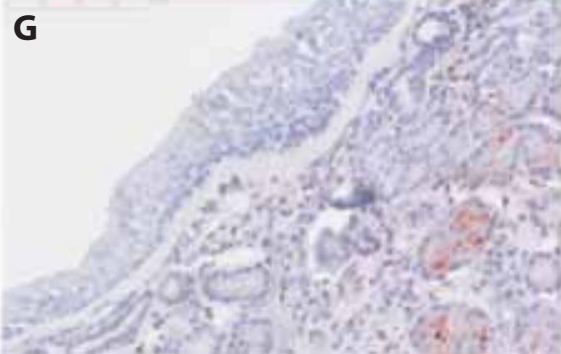

CRSwNP
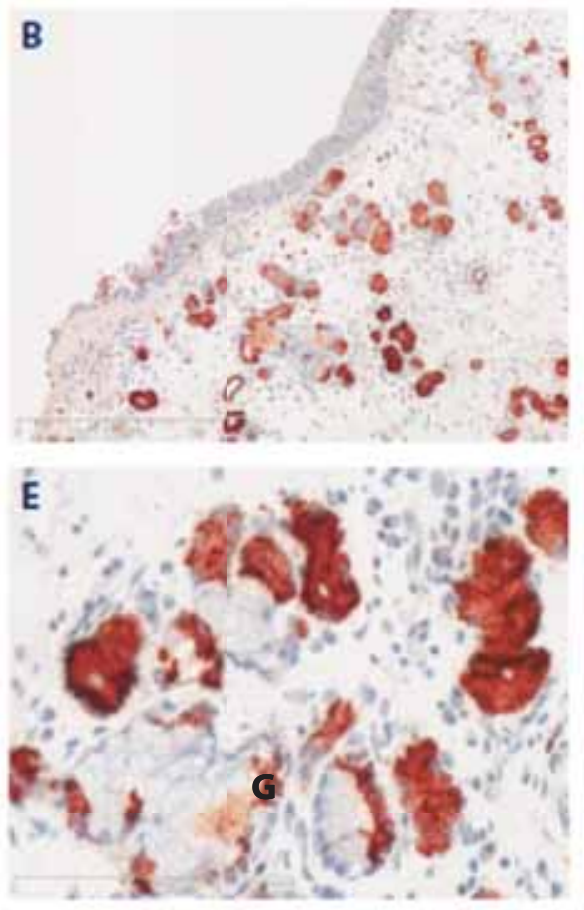

H

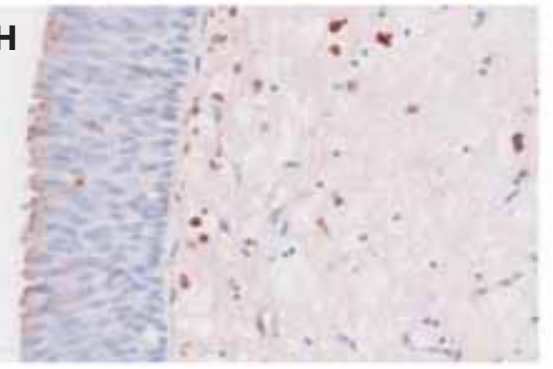

CRSsNP
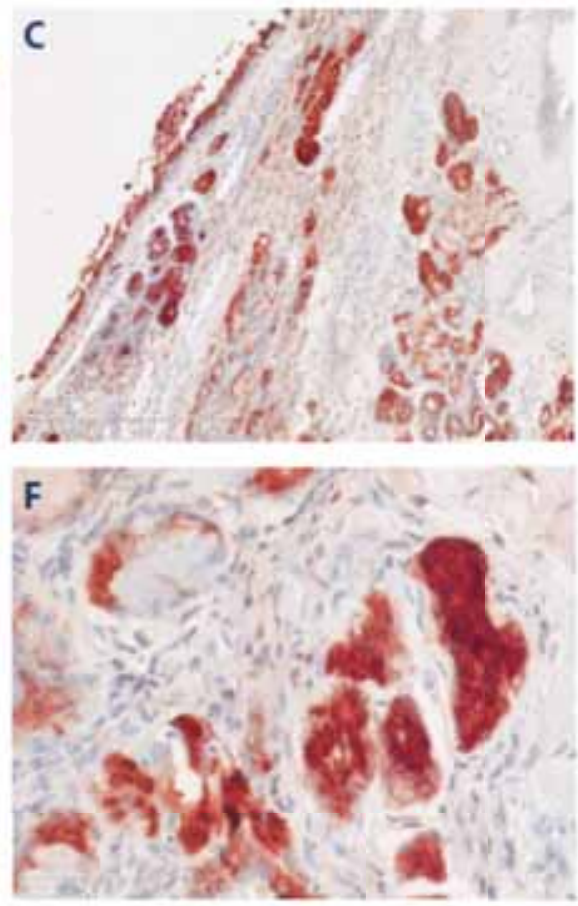

I

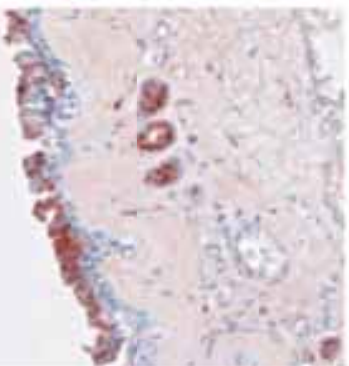

Figure 2. Distribution of lysozyme immunoreactivity in sinus mucosa of normal and CRS tissues.

Representative images of lysozyme-like immunoreactivity and distribution in seromucous glands and epithelium of normal ( $\mathrm{A}, \mathrm{D}, \mathrm{G}), \mathrm{CRSwNP}(\mathrm{B}, \mathrm{E}$, $H$ ) and CRSsNP (C, F, I) tissues. A, D, G: in normal tissue low level of lysozyme immunoreactivity was detected in some seromucous glands and epithelial cells. B, E, H: in CRSwNP strong lysozyme immunoreactivity was observed in many seromucous glands but at moderate levels in some epithelial cells. C, F, I: In CRSsNP strong lysozyme immunoreactivity was observed in many seromucous glands and also in some epithelial cells. Bar represents $400 \mathrm{~mm}$ in A, B and C. Bar represents $100 \mu \mathrm{m}$ in D-I.

Fungi have been implicated in the pathogenesis of CRS, but the significance of their involvement is debatable ${ }^{(35-41)}$. Our recent investigations demonstrated that lysozyme has fungicidal properties ${ }^{(9)}$ and this prompted us to determine if fungi were present in our CRS samples obtained during surgery. Interestingly, in our CRS patient population fungi were not identified histologically (using H\&E or PASD) on sinus mucosa biopsies, and rarely cultured from mucous obtained intra-operatively. Similarly, we did not identify the presence of fungi using a highly specific chitinase stain (Fungalase-F), despite positive detection of fungi in surgical sinus samples by other investiga- tors using this stain ${ }^{(42,43)}$. However, it is possible that the fungi, which reside in the mucus overlaying the epithelial cells were lost during routine formalin fixation of the sample. An alternative fixation technique utilizing Carnoy fluid to fix surface mucus in place has been successful for identifying bacteria in mucus of colon and adenoid biopsies ${ }^{(44)}$, and may prove useful in future studies for assessing microbial colonisation of sinus biopsies. The role of bacteria in promoting lysozyme secretion in the paranasal sinuses has not been studied in this paper and could be the subject of future study. The use of confocal microscopy and/or fluorescent in situ hybridization (FISH) techniques 
A

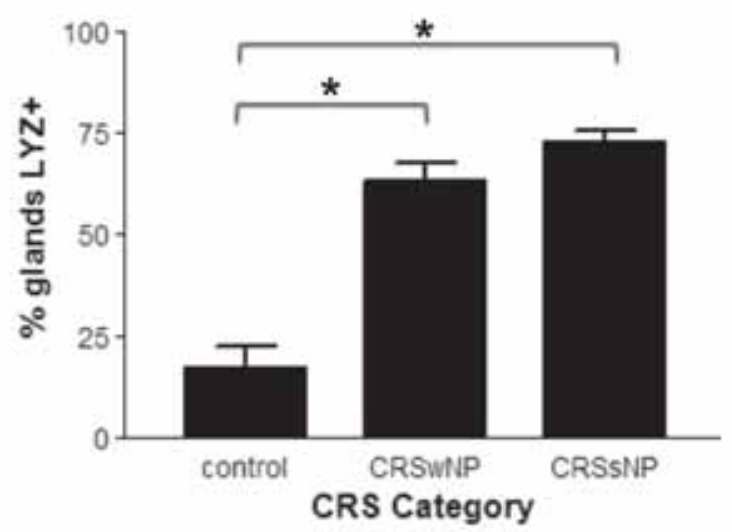

C

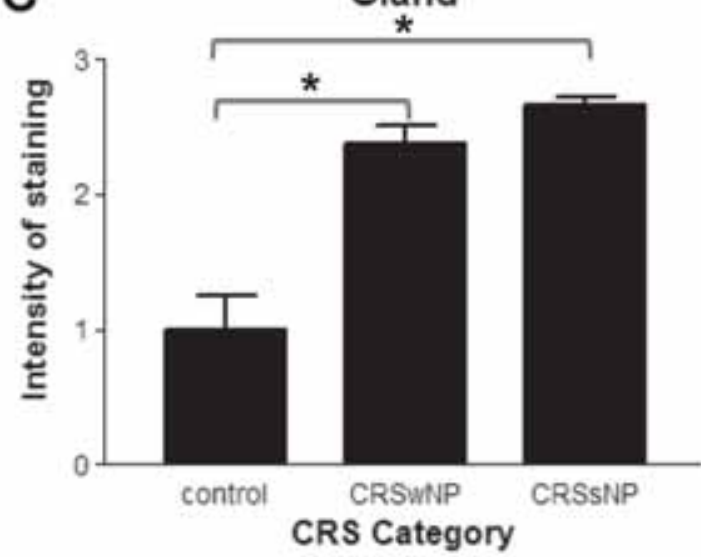

B Epithelium

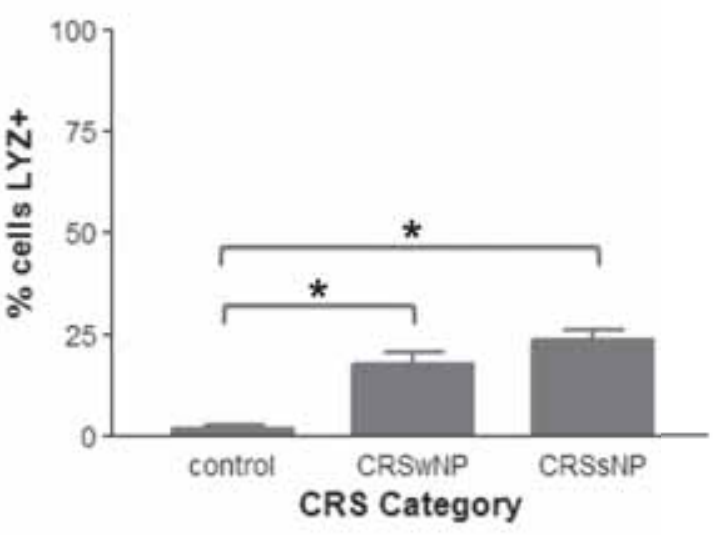

D Epithelium

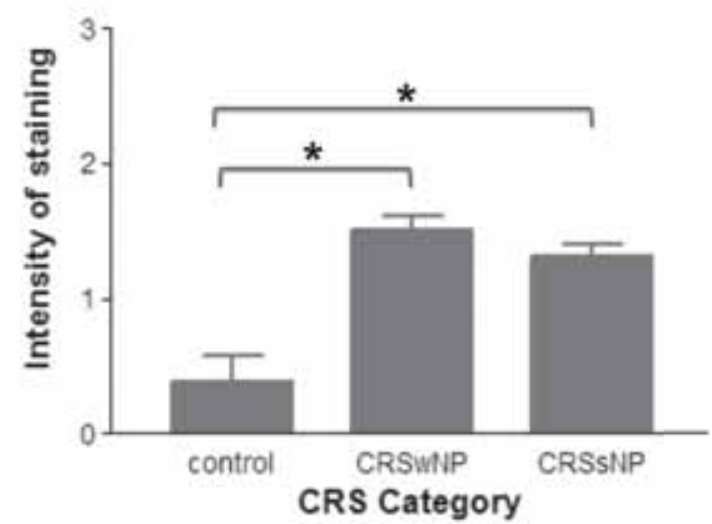

Figure 3. Higher levels of lysozyme immunoreactivity were found in the sinus mucosa of patients with CRS compared to normal sinus samples. Semi-quantitative analysis of lysozyme immunoreactivity observed in seromucous glands and epithelium of sinus mucosa specimens from control, CRS with nasal polyp (CRSwNP) and CRS without nasal polyp (CRSsNP). Lysozyme immunoreactivity is presented as a percentage of the number of seromucous glands (A) or epithelial cells (B). Intensity of lysozyme immunoreactivity scored on $1+, 2+3+$ scale for seromuous glands (C) and epithelium (D). Data presented as mean \pm SEM. $N=6$ control, 39 CRSwNP and 37 CRSsNP.

Lysozyme immunoreactivity was observed in a higher percentage of seromucous glands $(p<0.05)$ and epithelial cells $(p<0.05)$ in CRS specimens compared to normal sinus mucosa. The intensity of staining was also greater in the CRS specimens compared to normal samples ( $p<0.05$ ). There was no statistical difference in the level of lysozyme immunoreactivity between CRS categories. * denotes a significant difference between the two groups indicated.

has been useful for the detection of bacterial biofilms in sinus biopsies, but biofilms are not detected in every case of CRS ${ }^{(45)}$. Their detection in biopsies of normal healthy sinus tissue ${ }^{(46-48)}$ raises questions regarding the significance of biofilms in CRS, with the likely possibility that multiple factors are involved in the development of CRS. The role of dead fungi in stimulating the production of eosinophilic mucin is another area of research we are pursuing.

\section{Conclusion}

Lysozyme protein expression is increased in patients with CRS, predominantly in serous cells of submucosal glands. This data suggests that a deficiency in lysozyme protein expression is not likely responsible for the microbial colonisation often associated with CRS, and that the functional antimicrobial activity of lysozyme in the nasal secretions of these patients needs to be investigated further. 

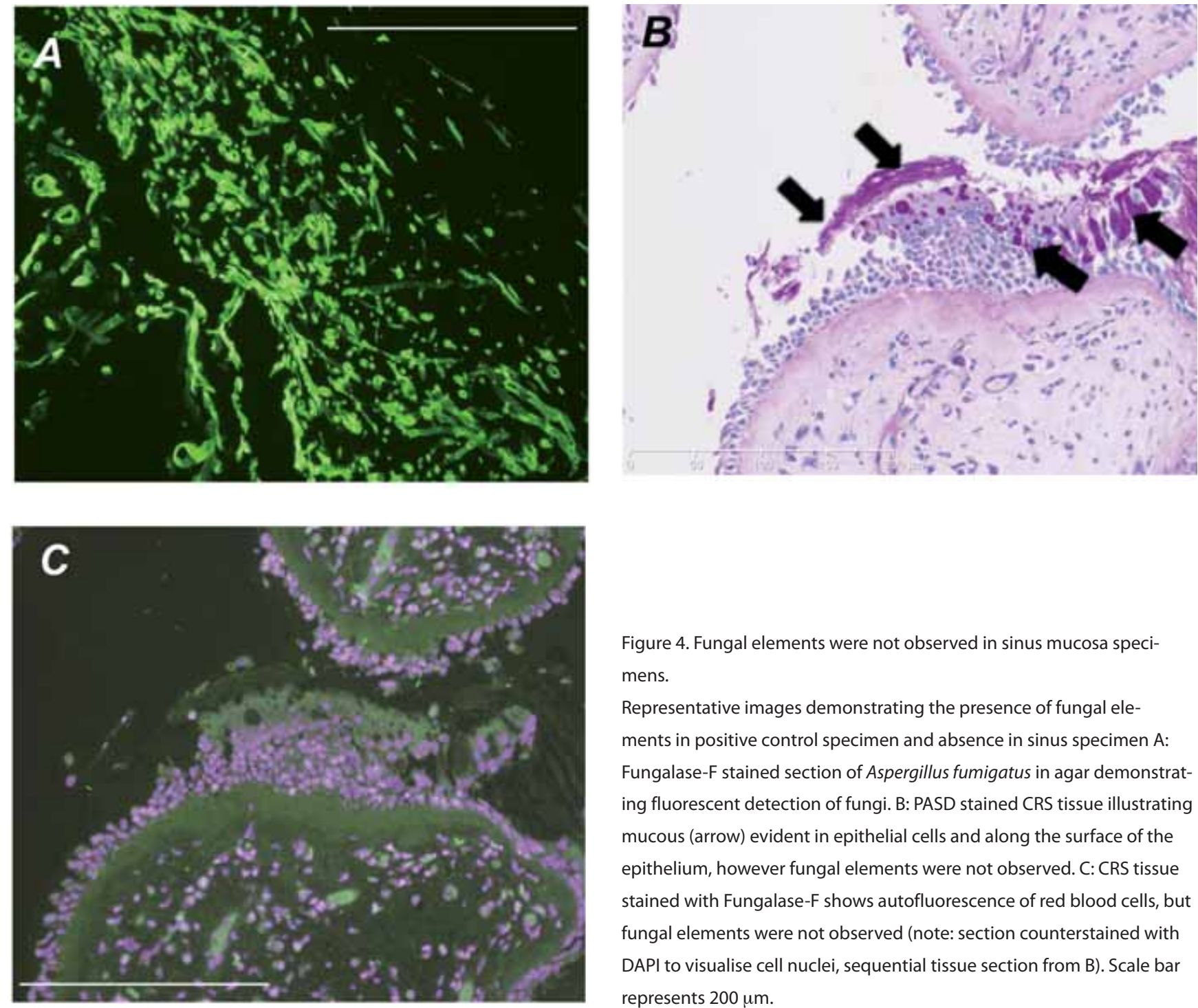

Figure 4. Fungal elements were not observed in sinus mucosa specimens.

Representative images demonstrating the presence of fungal elements in positive control specimen and absence in sinus specimen $\mathrm{A}$ : Fungalase-F stained section of Aspergillus fumigatus in agar demonstrating fluorescent detection of fungi. B: PASD stained CRS tissue illustrating mucous (arrow) evident in epithelial cells and along the surface of the epithelium, however fungal elements were not observed. C: CRS tissue stained with Fungalase-F shows autofluorescence of red blood cells, but fungal elements were not observed (note: section counterstained with DAPI to visualise cell nuclei, sequential tissue section from B). Scale bar represents $200 \mu \mathrm{m}$.

Table 1. Primer sequences and PCR product size.

$\begin{array}{ccc}\text { Primer Name } & \text { Primer sequence } & \text { Amplicon Size (bp) } \\ \text { LYZ F } & \text { GCATGGAGAAATCGTTGTCA } & 196 \\ \text { LYZ R } & \text { GCCATATTTTGCTCCTGCTT } & 93 \\ \text { HPRT F } & \text { TGACACTGGCAAAACAATGCA } & \\ \text { HPRT R } & \text { GGTCCTTTTCACCAGCAAGCT }\end{array}$

\section{Acknowledgements}

The authors are grateful for the support provided by Professor PJ Wormald, and the Garnett Passe and Rodney Williams Memorial foundation for funding this study.

\section{Authorship contribution}

CW: experimental design, data acquisition, data analysis, manuscript preparation. VL: data acquisition, manuscript prepara- tion. DH: experimental design, data analysis, manuscript preparation. SI: data analysis, manuscript preparation. EO: funding of project, data review, manuscript preparation. LT: funding of project, data review, manuscript preparation. ASC: funding of project, data analysis, manuscript preparation.

\section{Conflict of interest}

The authors have no conflict of interests related to this study. 


\section{References}

1. Ramanathan M, Jr., Lane AP. Innate immunity of the sinonasal cavity and its role in chronic rhinosinusitis. Otolaryngol Head Neck Surg. 2007; 136: 348-356.

2. Ooi EH, Wormald P-J, Tan LW. Innate immunity in the paranasal sinuses: a review of nasal host defenses. American Journal of Rhinology. 2008; 22: 13-19.

3. Ooi E, Psaltis A, Witterick I, Wormald P-J. Innate immunity. The Otolaryngologic clinics of North America. 2010; 43: 473-487,vii.

4. Cole AM, Liao HI, Stuchlik O, Tilan J, Pohl J, Ganz T. Cationic polypeptides are required for antibacterial activity of human airway fluid. J Immunol. 2002; 169: 6985-6991.

5. Kirby AJ. The lysozyme mechanism sorted -- after 50 years. Nat Struct Biol. 2001; 8: 737-739.

6. Ibrahim HR, Matsuzaki T, Aoki T. Genetic evidence that antibacterial activity of lysozyme is independent of its catalytic function. FEBS letters. 2001; 506: 27-32.

7. Laible NJ, Germaine GR. Bactericidal activity of human lysozyme, muramidaseinactive lysozyme, and cationic polypeptides against Streptococcus sanguis and Streptococcus faecalis: inhibition by chitin oligosaccharides. Infection and immunity. 1985; 48: 720-728.

8. Masschalck B, Deckers D, Michiels CW. Lytic and nonlytic mechanism of inactivation of gram-positive bacteria by lysozyme under atmospheric and high hydrostatic pressure. J Food Prot. 2002; 65: 1916-1923.

9. Woods CM, Hooper DN, Ooi EH, Tan LW, Carney AS. Human lysozyme has fungicidal activity against nasal fungi. Am J Rhin Allergy. 2011; 25: 236-240.

10. Hornef MW, Wick MJ, Rhen M, Normark S. Bacterial strategies for overcoming host innate and adaptive immune responses. Nat Immunol. 2002; 3: 1033-1040.

11. Ganz T. Fatal attraction evaded. How pathogenic bacteria resist cationic polypeptides. J Exp Med. 2001; 193: F31-34.

12. Samaranayake $Y H$, Samaranayake LP, Pow EH, Beena VT, Yeung KW. Antifungal effects of lysozyme and lactoferrin against genetically similar, sequential Candida albicans isolates from a human immunodeficiency virus-infected southern Chinese cohort. J Clin Microbiol. 2001; 39: 3296-3302.

13. Fukami M, Stierna P, Veress B, Carlsoo B. Lysozyme and lactoferrin in human maxillary sinus mucosa during chronic sinusitis. An immunohistochemical study. Eur Arch Otorhinolaryngol. 1993; 250: 133-139.

14. Kim SS, Kim KS, Lee JG, Park IY, Koo JS, Yoon JH. Levels of intracellular protein and messenger RNA of mucin and lysozyme in normal human nasal and polyp epithelium. Laryngoscope. 2000; 110 (2 Pt 1): 276-280.

15. Raphael GD, Jeney EV, Baraniuk JN, Kim I, Meredith SD, Kaliner MA. Pathophysiology of rhinitis. Lactoferrin and lysozyme in nasal secretions. J Clin Invest. 1989; 84: 15281535
16. Tachibana M, Morioka H, Machino $M$, Tsuruoka T, Tanimura F, Mizukoshi O Lysozyme producers in nasal mucosa. An immunocytochemical study. Ann Otol Rhinol Laryngol. 1986; 95 (2 Pt 1): 193-195.

17. Meltzer EO, Hamilos DL, Hadley JA, et al. Rhinosinusitis: Establishing definitions for clinical research and patient care. Otolaryngology--head and neck surgery. 2004; 131 (6 Suppl): S1-62.

18. Ooi EH, Wormald PJ, Carney AS, James CL, Tan LW. Fungal allergens induce cathelicidin LL-37 expression in chronic rhinosinusitis patients in a nasal explant model. Am J Rhinol. 2007; 21: 367-372.

19. Ooi EH, Wormald PJ, Carney AS, James CL, Tan LW. Surfactant protein to d expression in chronic rhinosinusitis patients and immune responses in vitro to Aspergillus and Alternaria in a nasal explant model. Laryngoscope. 2007; 117: 51-57.

20. Ooi EH, Wormald PJ, Carney AS, James CL, Tan LW. Human cathelicidin antimicrobial peptide is up-regulated in the eosinophilic mucus subgroup of chronic rhinosinusitis patients. Am J Rhinol. 2007; 21: 395-401.

21. Simon P. Q-Gene: processing quantitative real-time RT-PCR data. Bioinformatics. 2003; 19: 1439-1440.

22. Fahlgren $A$, Hammarstrom $S$, Danielsson $A$ Hammarstrom ML. Increased expression of antimicrobial peptides and lysozyme in colonic epithelial cells of patients with ulcerative colitis. Clin Exp Immunol. 2003: 131: 90-101.

23. Lei W, Jaramillo RJ, Harrod KS. Transactivation of lung lysozyme expression by Ets family member ESE-1. Am J Physiol Lung Cell Mol Physiol. 2007; 293: L1359-1368.

24. Yoon JH, Kim KS, Kim SS, Lee JG, Park IY. Secretory differentiation of serially passaged normal human nasal epithelial cells by retinoic acid: expression of mucin and lysozyme. Ann Otol Rhinol Laryngol. 2000; 109: 594-601.

25. Yoon JH, Moon HJ, Seong JK, et al. Mucociliary differentiation according to time in human nasal epithelial cell culture. Differentiation. 2002; 70: 77-83.

26. Machino M, Morioka $H$, Tachibana M, Tsuruoka T, Mizukoshi O. Studies of the substructures of the lysozyme-rich secretory granule of the serous cell in the human nasal gland. Arch Histol Jpn. 1984; 47: 549551.

27. Filipowicz W, Bhattacharyya SN, Sonenberg N. Mechanisms of post-transcriptional regulation by microRNAs: are the answers in sight? Nat Rev Genet. 2008; 9: 102-114.

28. O'Neill LA, Sheedy FJ, McCoy CE. MicroRNAs: the fine-tuners of Toll-like receptor signalling. Nat Rev Immunol. 2011; 11: 163-175.

29. Dai R, Ahmed SA. MicroRNA, a new paradigm for understanding immunoregulation, inflammation, and autoimmune diseases. Transl Res. 2011; 157: 163-179.
30. Wang $X$, EI Naqa IM. Prediction of both conserved and nonconserved microRNA targets in animals. Bioinformatics. 2008; 24: 325-332.

31. Singh PK, Tack BF, McCray PB, Jr., Welsh MJ. Synergistic and additive killing by antimicrobial factors found in human airway surface liquid. Am J Physiol Lung Cell Mol Physiol. 2000; 279: L799-805.

32. Travis SM, Conway BA, Zabner J, et al. Activity of abundant antimicrobials of the human airway. Am J Respir Cell Mol Biol. 1999; 20: 872-879

33. Joris L, Dab I, Quinton PM. Elemental composition of human airway surface fluid in healthy and diseased airways. Am Rev Respir Dis. 1993: 148 (6 Pt 1): 1633-1637.

34. Knowles MR, Robinson JM, Wood RE, et al. Ion composition of airway surface liquid of patients with cystic fibrosis as compared with normal and disease-control subjects. J Clin Invest. 1997; 100 : 2588-2595.

35. Orlandi RR, Marple BF. The role of fungus in chronic rhinosinusitis. Otolaryngologic Clinics of North America. 2010; 43: 531-537.

36. Orlandi RR, Marple BF. Fungus and chronic rhinosinusitis: weighing the evidence. Otolaryngology - Head \& Neck Surgery. 2010; 143: 611-613.

37. Ponikau JU, Sherris DA, Kern EB. Immunologic response to fungus is not universally associated with chronic rhinosinusitis. Otolaryngology - Head \& Neck Surgery. 2010; 143: 607-610.

38. Hamilos DL. Allergic fungal rhinitis and rhinosinusitis. Proc of the American Thoracic Society. 2010; 7: 245-252.

39. Fokkens WJ, Ebbens F, van Drunen CM. Fungus: a role in pathophysiology of chronic rhinosinusitis, disease modifier, a treatment target, or no role at all? Immunology \& Allergy Clinics of North America. 2009; 29: 677-688.

40. Pant H, Schembri MA, Wormald PJ, Macardle PJ. IgE-mediated fungal allergy in allergic fungal sinusitis. Laryngoscope. 2009; 119 (6):1046-1052.

41. Rank MA, Adolphson CR, Kita H. Antifungal therapy for chronic rhinosinusitis: the controversy persists. Current Opinion in Allergy \& Clinical Immunology. 2009; 9: 67-72.

42. Taylor MJ, Ponikau JU, Sherris DA, et al. Detection of fungal organisms in eosinophilic mucin using a fluoresceinlabeled chitin-specific binding protein. Otolaryngology--head and neck surgery. 2002; 127: 377-383.

43. Bassiouny A, Ragab A, Attia AF, et al Prevalence of extramucosal fungal elements in sinonasal polyposis: a mycological and pathologic study in an Egyptian population. Am J Otolaryngol. 2011; 32: 308-317.

44. Winther B, Gross BC, Hendley JO, Early SV. Location of bacterial biofilm in the mucus overlying the adenoid by light microscopy. Arch Otolaryngol Head Neck Surg. 2009; 135: 1239-1245. 
45. Keir J, Pedelty L, Swift AC. Biofilms in chronic rhinosinusitis: systematic review and suggestions for future research. J Laryngol Otol. 2011; 125: 331-337.

46. Sanderson AR, Leid JG, Hunsaker D. Bacterial biofilms on the sinus mucosa of human subjects with chronic rhinosinusitis. Laryngoscope. 2006; 116: 1121-1126.

47. Healy DY, Leid JG, Sanderson AR, Hunsaker $\mathrm{DH}$. Biofilms with fungi in chronic rhinosinusitis. Otolaryngol Head Neck Surg. 2008; 138: 641-647.

48. Bezerra TF, Padua FG, Gebrim EM, Saldiva $\mathrm{PH}$, Voegels RL. Biofilms in chronic rhinosinusitis with nasal polyps. Otolaryngol Head Neck Surg. 2011; 144: 612-616.

\section{Professor A. Simon Carney}

Professor of Otolaryngology - Head

\& Neck Surgery

Head of Unit

Flinders ENT

Department of Surgery

Flinders Medical Centre and Flinders

University

\section{Mail: Suite 200}

Flinders Private Hospital

Flinders Drive

Bedford Park, SA 5042

AUSTRALIA

Tel: +61-8-8204 6187

Fax: +61-8-8204 3987

E-mail: scarney@ent-surgery.com

\section{ADVERTISEMENT}

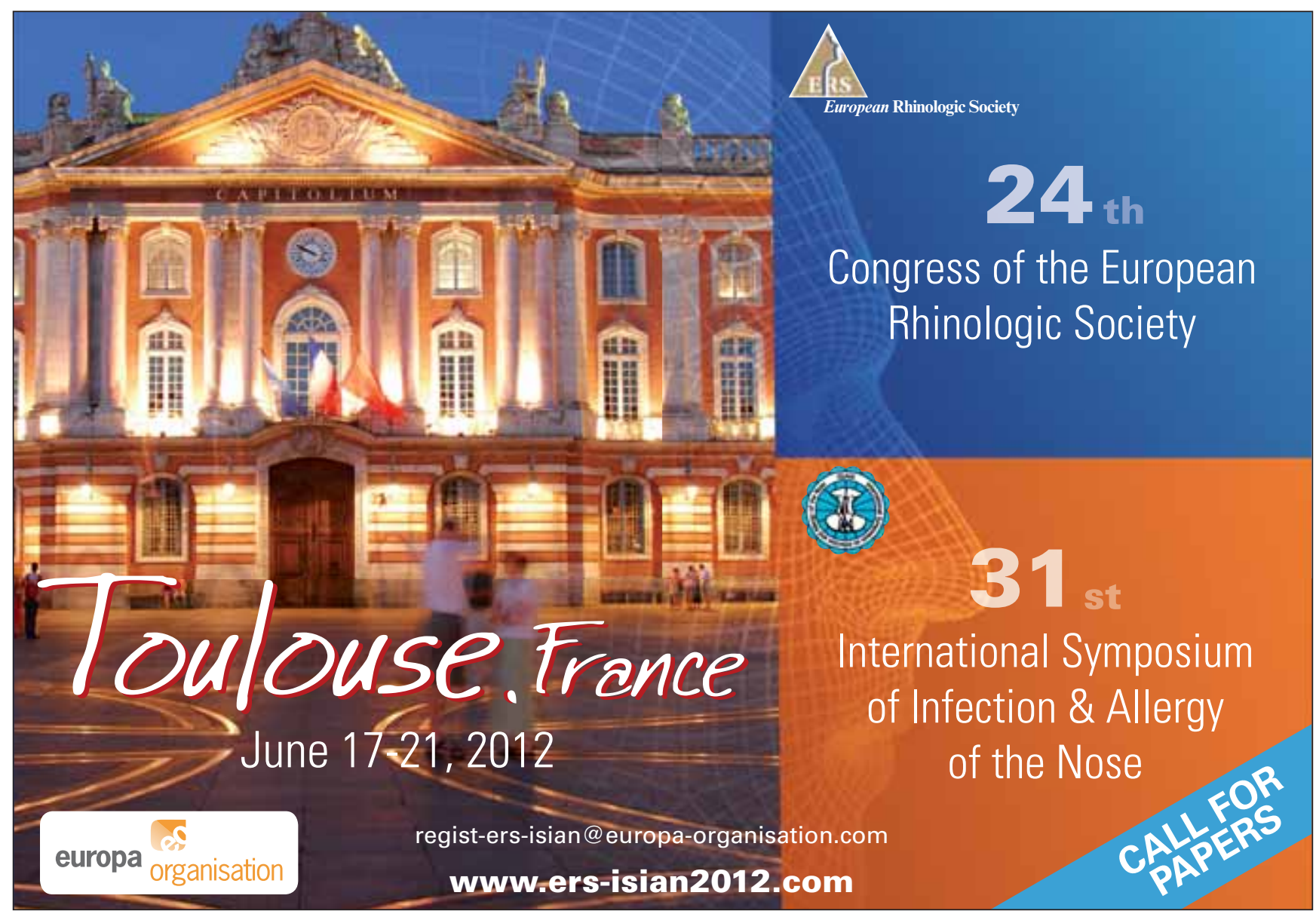

